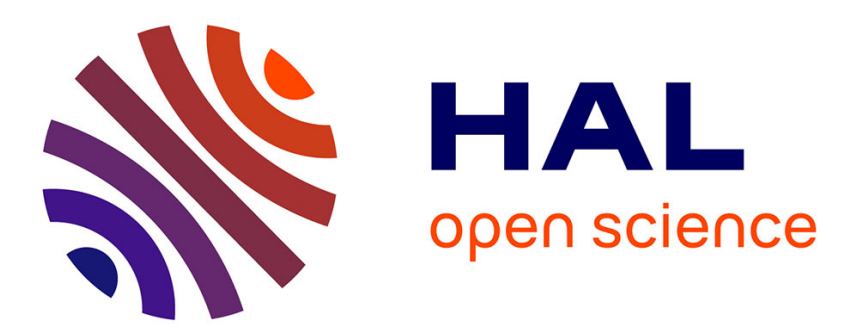

\title{
Association de betteraves fourragères à une ration mixte d'ensilages de maïs et de trèfle violet pour vaches laitières
}

\author{
A. Hoden, B. Marquis, Luc Delaby
}

\section{> To cite this version:}

A. Hoden, B. Marquis, Luc Delaby. Association de betteraves fourragères à une ration mixte d'ensilages de maïs et de trèfle violet pour vaches laitières. Productions Animales, 1988, 1 (3), pp.165169. hal-00895828

\section{HAL Id: hal-00895828 https://hal.science/hal-00895828}

Submitted on 1 Jan 1988

HAL is a multi-disciplinary open access archive for the deposit and dissemination of scientific research documents, whether they are published or not. The documents may come from teaching and research institutions in France or abroad, or from public or private research centers.
L'archive ouverte pluridisciplinaire HAL, est destinée au dépôt et à la diffusion de documents scientifiques de niveau recherche, publiés ou non, émanant des établissements d'enseignement et de recherche français ou étrangers, des laboratoires publics ou privés. 
INRA Prod. Anim., 1988, 1 (3), 165-169

\section{A. HODEN, B. MARQUIS \\ L. $D E L A B Y^{\star}$}

INRA Saint-Gilles

Station de Recherches sur la Vache Laitière 35590 L'Hermitage

* INRA Nouzilly

Station de Physiologie de la Reproduction 37380 Monnaie

\section{Association de betteraves fourragères à une ration mixte d'ensilages de maïs et de trèfle violet pour vaches laitières}

Les rations mixtes à base d'ensilages de maïs et de trèfle violet distribuées aux vaches laitières entraînent des diminutions de productions de « matière utile » du lait et de sa composition par rapport à l'ensilage de maïs seul. L'association de la betterave fourragère à ce type de régime peut-elle corriger de tels effets dépressifs?

\begin{abstract}
Depuis une dizaine d'années, les surfaces de betteraves fourragères ont augmenté dans les régions défavorables à la production de l'ensilage de maïs telles que les zones les plus sèches et surtout les plus froides de Bretagne (Kerouanton 1982 et 1988) où les rendements du maïs planteentière varient dans de larges proportions (de 1 à 2) selon les conditions climatiques de l'année. Les mêmes raisons expliquent aussi le développement des cultures telles que le trèfle violet afin de disposer d'un aliment de « sécurité » permettant d'alimenter correctement les troupeaux de vaches laitières. Mais ces rations mixtes à base d'ensilages de maïs et de trèfle présentent certains inconvénients par rapport au régime d'ensilage de maïs seul puisqu'elles entraînent des diminutions de quantités de matière utile et surtout de protéines secrétées ainsi qu'une réduction de la notation de l'état d'engraissement des animaux (Espinasse 1986, Hoden et al 1987, Dedenon et al 1988).
\end{abstract}

\section{Résumé}

Des betteraves fourragères entières ont été distribuées $(20 \mathrm{~kg} / \mathrm{j})$ en complément d'une ration mixte d'ensilages de maïs et de trèfle violet afin de mettre en évidence leurs effets sur les performances des vaches laitières. Cette association a permis d'améliorer les productions de lait $(+2 \mathrm{~kg} / \mathrm{j})$ et de matière utile $(+100 \mathrm{~g} / \mathrm{j}$ matières grasses, $+60 \mathrm{~g} / \mathrm{j}$ matières protéiques) ainsi que la composition du lait (taux butyreux : $+2 \mathrm{~g} / \mathrm{kg}$, taux protéique $:+1 \mathrm{~g} / \mathrm{kg}$ ). Ces effets positifs sont probablement à attribuer aux modifications d'orientations fermentaires dans le rumen concernant le taux butyreux et au meilleur niveau d'apport énergétique pour la synthèse des protéines.

Dans nos conditions d'utilisation, ces betteraves apportées en quantités relativement faibles ont permis d'augmenter le niveau de production permis par la ration de base d'environ $2,5 \mathrm{~kg}$ de lait. Leur valorisation énergétique correspond à celle proposée par les nouvelles Tables INRA (1988).
L'apport de betteraves fourragères devrait parfaitement se justifier avec de telles rations de base pour corriger ces effets dépressifs. En plus de leur apport d'énergie, favorable à la synthèse de protéines (Rémond 1985), les betteraves présentent en effet l'avantage de favoriser la reprise de poids et d'améliorer le taux butyreux en raison des orientations fermentaires qu'elles provoquent au sein du rumen (Vérité et Journet 1973, Journet et Chilliard 1985).

Un essai a été mis en place à l'INRA de Rennes en 1987, en liaison avec l'ADBFM (Association pour le Développement de la Betterave Fourragère Monogerme), afin de mettre en évidence les effets de l'apport de betteraves fourragères en complément d'une ration mixte à base d'ensilages de maïs et de trèfle violet sur les performances des vaches laitières.

\section{Principales conditions expérimentales}

28 vaches laitières de type Holstein ont été réparties en deux lots comparables de 14 vaches, appariées au cours d'une période pré-expérimentale de 3 semaines située durant la phase croissante de la production (tableau 1). La période d'essai proprement dite de 13 semaines a débuté en moyenne au pic de la lactation et s'est terminée à la mise à l'herbe des vaches. Les 3 premières semaines de pâturage, consécutives à l'essai, ont servi de période post-expérimentale. Les conditions expérimentales sont détaillées dans le tableau 1.

Les deux lots témoin et expérimental de vaches laitières ont reçu une même ration composée d'ensilage de maïs apporté en quantité limitée (environ $6 \mathrm{~kg} \mathrm{MS}$ ) et d'ensilage de trèfle 
Tableau 1.

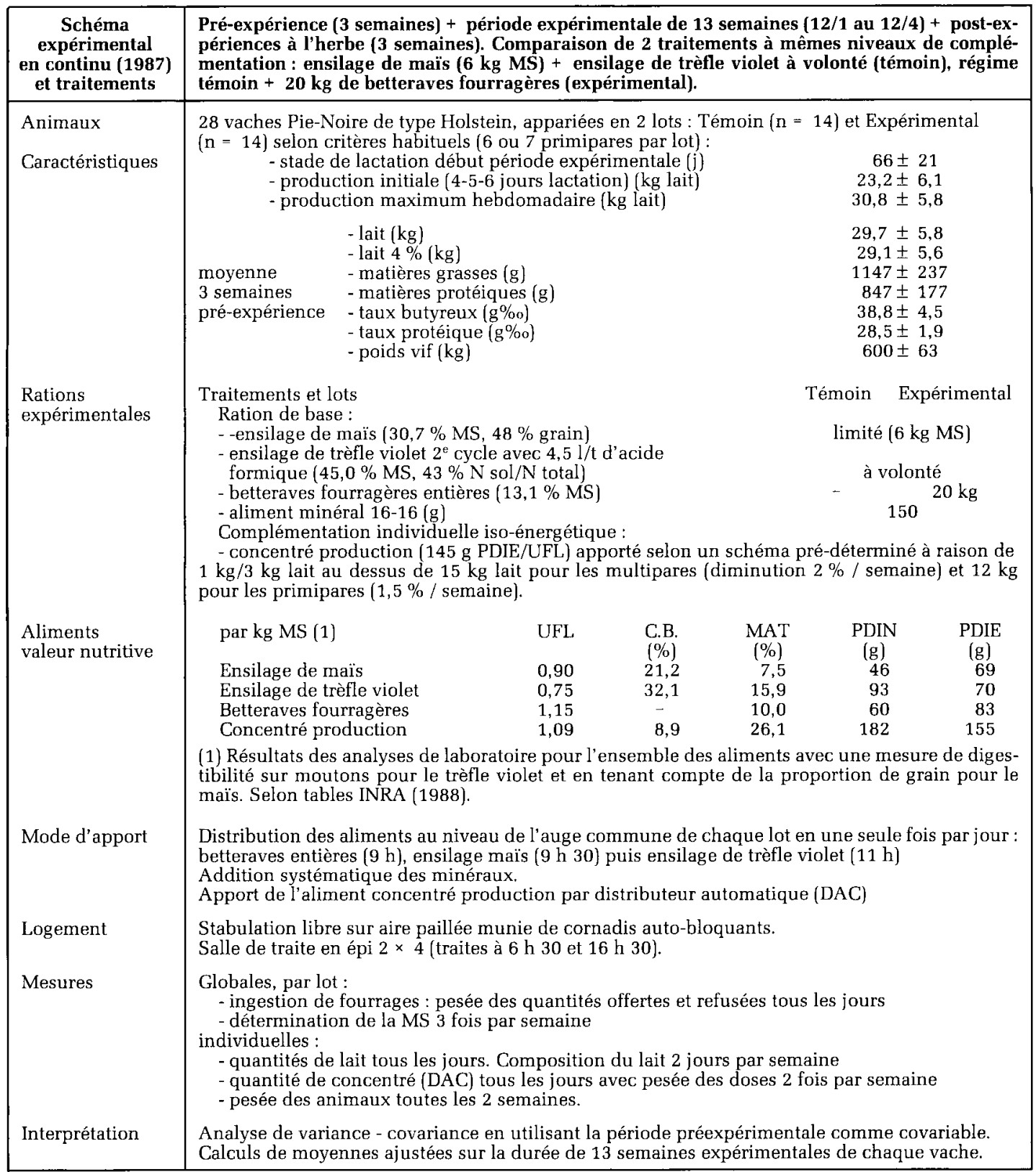

violet de bonne qualité ( $2^{\mathrm{e}}$ cycle, préfané à $45 \%$ MS) distribué à volonté. De plus, le lot expérimental (" betteraves ") a reçu une ration journalière de $20 \mathrm{~kg}$ de betteraves entières (13\% MS) apportées le matin comme seul aliment avant la distribution de l'ensilage de maïs.

Une même complémentation iso-énergétique et azotée a été utilisée au niveau de chacun des régimes supposés équilibrés entre les UFL et les PDI en apportant de l'aliment concentré ( $1 \mathrm{~kg} /$ $3 \mathrm{~kg}$ lait) au-dessus de $15 \mathrm{~kg}$ de lait $4 \%$ (12 kg pour les primipares] selon un schéma prédéterminé.

\section{Résultats}

L'association de $20 \mathrm{~kg}$ de betteraves fourragères entières $(2,5 \mathrm{~kg} \mathrm{MS})$ à la ration témoin d'ensilages de maïs et de trèfle violet s'est traduite par (tableau 2, figure 1) :

\section{un léger accroissement des quantités ingérées et des apports énergétiques}

Les niveaux d'ingestion de la ration de base ont été en moyenne d'environ 2,3 kg MS par 100 $\mathrm{kg}$ de poids vif ; ils correspondent à ceux habituellement obtenus avec des fourrages de bonne qualité (Hoden et al 1988). L'ensilage de trèfle violet a représenté $55 \%$ de la MS de la ration de base avec le régime témoin et $39 \%$ avec la ration expérimentale. Les betteraves ont alors représenté $17 \%$ de la ration de base et $14 \%$ de la MS totale. L'apport de 2,5 kg MS de betteraves a entraîné une diminution de la consommation d'ensilage de trèfle violet de $2 \mathrm{~kg}$ MS soit un taux de substitution marginal d'environ 0,85 compte tenu de l'apport légèrement inférieur [0,2 kg MS $)$ d'aliment concentré avec le régime expérimental. Dans ces conditions, le niveau de la ration de base n'a été supérieur que de $0,5 \mathrm{~kg}$ MS avec la ration "betteraves » $(14,3$ contre $13,8 \mathrm{~kg} \mathrm{MS}$ ) et celui de la ration totale de $0,3 \mathrm{~kg}$ MS (17,7 contre 17,4 kg MS). Simultanément, 
les apports énergétiques réels (INRA 1988) de la ration « betteraves » ont été supérieurs d'environ 1 UFL à ceux du régime témoin et les quantités de PDI ont été identiques (1550 g environ) avec cependant un léger excès d'apport d'azote dégradable avec la ration témoin $(+100 \mathrm{~g}$ PDIN).

\section{une amélioration des performances}

Les productions journalières de lait $4 \%$, de matières grasses et protéiques ont été plus élevées $(\mathrm{P}<0,01)$ avec le régime comportant des betteraves : $+2,0 \mathrm{~kg},+100 \mathrm{~g}$ et $+60 \mathrm{~g}$ respectivement par rapport au régime témoin. Simultanément, le taux butyreux a été supérieur de $2 \mathrm{~g}$ par $\mathrm{kg}(\mathrm{P}<0,05)$ et celui de protéines de $1 \mathrm{~g}$ par kg. Durant la période expérimentale (13 semaines), les persistances mensuelles de la production laitière brute ont été respectivement de $91 \%$ et $93,5 \%$ pour les régimes témoin et expérimental.

A la mise à l'herbe, les différentes variables de production (lait, matière utile) et la composition du lait (taux butyreux et protéique) se sont toutes améliorées et sont généralement redevenues à des valeurs identiques (sauf dans le cas du taux butyreux) pour les 2 lots.

Les poids vifs des animaux des 2 lots sont sensiblement restés constants durant la période expérimentale. En supposant que les 2 lots avaient des contenus digestifs identiques en pré et post-
Figure 1. Evolution de quelques variables de production et de la composition du lait au cours de l'essai.
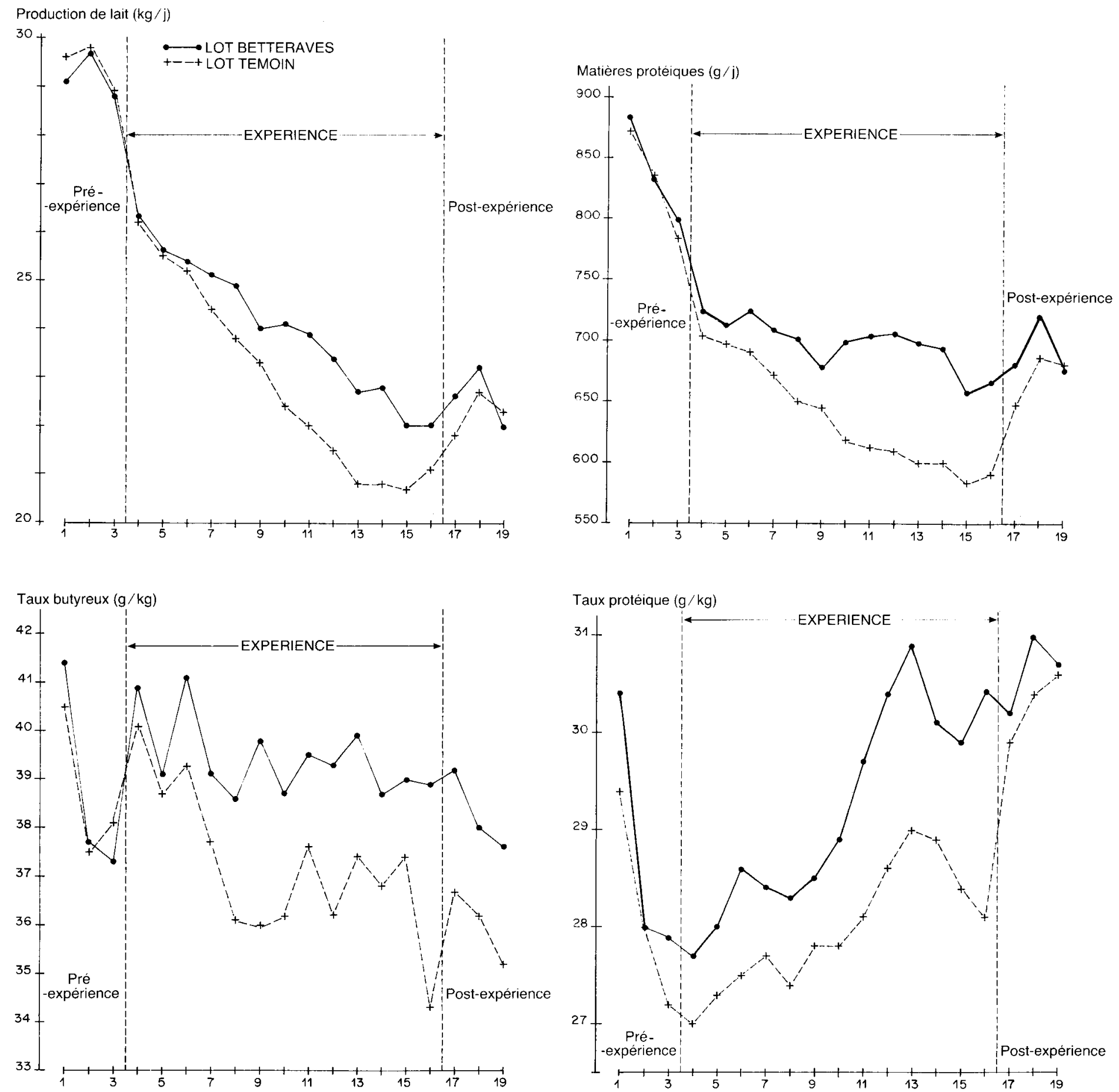
expériences, les vaches du lot « betteraves » auraient perdu $10 \mathrm{~kg}$ de moins que celles du lot témoin soit une variation de poids vif d'environ $80 \mathrm{~g} / \mathrm{j}$ durant l'essai.

\section{une valorisation normale de l'énergie apportée}

Après avoir tenu compte des interactions liées à l'utilisation de l'énergie (Faverdin et al 1987), les bilans énergétiques sont voisins de l'équilibre (+ 0,1 ̀̀ + 0,2 UFL) pour les vaches des 2 traitements. De même, les besoins en PDI sont satisfaits. Ces bilans n'intègrent pas les variations de poids vif apparentes observées durant l'essai entre les vaches des 2 régimes.

Les apports énergétiques de la ration de base, estimés à partir des performances de production des animaux (besoins production laitière - apports concentrés), auraient permis 13,0 et 15,5 $\mathrm{kg}$ de lait $4 \%$ respectivement pour les rations " témoin » et « betteraves ». Les valorisations énergétiques de ces rations (besoins totaux $/ \mathrm{kg}$ MST) ont correspondu à celles attendues par les valeurs nutritives des rations (apports totaux réels/kg MST).

\section{Discussion / Conclusion}

Les résultats de cet essai ont permis de confirmer ou de préciser différents aspects se rapportant à l'utilisation de la ration mixte d'ensilages de maïs + trèfle violet associée ou non à des betteraves.

- Le régime témoin, identique à celui utilisé précédemment (Hoden et al 1987), mèt à nouveau en évidence l'influence négative de la ration de base mixte avec du trèfle violet sur les performances de production et de composition du lait. Les taux butyreux $(37,3 \mathrm{~g} \%$ ) et protéique $(28,0$ $\mathrm{g} \%$ o) sont en particulier anormalement bas par rapport à ceux observés avec des rations composées uniquement d'ensilage de maïs (Hoden et al 1985). Le stade de lactation des animaux $\left(2^{e_{-}}\right.$ $3^{\mathrm{e}}$ mois) peut en partie expliquer ce faible taux protéique (Rémond 1984).

- L'introduction de betteraves à cette ration mixte a permis d'améliorer significativement les productions de lait et de matière utile ainsi que le taux butyreux. Cette association a ainsi permis d'obtenir une réponse marginale de $0,8 \mathrm{~kg}$ de lait $4 \%$ par $\mathrm{kg}$ de MS de betteraves. Le taux protéique demeure cependant encore à un niveau insuffisant $(29 \mathrm{~g} \%$ ) tant pour l'éleveur (paiement du lait) que pour le fromager (Kerjean 1988). Les effets bénéfiques de l'introduction supplémentaire de betteraves sont vraisemblablement à attribuer d'une part à des modifications d'orientations fermentaires dans le rumen (acide butyrique) favorables à la synthèse des matières grasses (Journet et Chilliard 1985) et d'autre part au meilleur niveau d'apport énergétique pour la synthèse des protéines (Rémond 1985).

Tableau 2. Principaux résultats expérimentaux. Moyennes des 13 semaines expérimentales.

\begin{tabular}{|c|c|c|c|}
\hline Lots & Témoin & Expérimental & \\
\hline - Effectif & 14 & 14 & \\
\hline - Quantités ingérées (kg MS) & & & \\
\hline Ensilage de maïs & 6,2 & 6,2 & \\
\hline Ensilage de trèfle violet & 7,6 & 5,6 & \\
\hline Betteraves & - & 2,5 & \\
\hline Ration de base par $100 \mathrm{~kg}$ poids vif & 2,28 & 2,36 & \\
\hline Aliment concentré production & 3,6 & 3,4 & \\
\hline Ration totale par $100 \mathrm{~kg}$ poids vif & 2,88 & 2,92 & \\
\hline - Apports et bilans nutritifs ( 1 ) & & & \\
\hline UFL (2) & 14,7 & 15,7 & \\
\hline PDIN (g) & 1645 & 1575 & \\
\hline PDIE (g) & 1520 & 1555 & \\
\hline Bilan énergétique - UFL (2) & 0,1 & 0,2 & \\
\hline Bilan PDI (g) & 75 & 15 & \\
\hline $\mathrm{MAT}(\% \mathrm{MS})$ & 15,4 & 14,4 & \\
\hline - Performances - moyennes ajustées & & & ETR \\
\hline Lait (kg) & 22,7 & 24,1 & 2,2 \\
\hline Lait $4 \%(\mathrm{~kg})$ & 21,8 & 23,8 & $1,4^{* *}$ \\
\hline Matières grasses (g) & 846 & 946 & $60^{* *}$ \\
\hline Matières protéiques $(\mathrm{g})$ & 636 & 696 & $53^{* *}$ \\
\hline Taux butyreux (g \%o) & 37,3 & 39,3 & $2,6^{*}$ \\
\hline Taux protéique $(\mathrm{g} \% \mathrm{c}$ ] & 28,0 & 29,0 & 1,7 \\
\hline Poids vif (kg) & 604 & 606 & 13 \\
\hline Variations de poids vif $(3)(\mathrm{kg})$ & -50 & -40 & \\
\hline
\end{tabular}

ETR - Ecart type résiduel - * $\mathrm{P}<0,05 ;{ }^{*} \mathrm{P}<0,01$

(1) Selon Tables INRA (1988)

(2) Y compris corrections de la valeur énergétique de la ration (INRA 1988)

(3) Différences entre les 3 semaines des 2 périodes pré et post-expérimentales (régime hivernal - herbe) 
- A même niveau de complémentation, l'introduction de betteraves (2,5 kg MS) a entraîné une diminution importante de l'ingestion du fourrage offert à volonté (ensilage de trèfle violet) puisque le taux de substitution marginal a été d'environ 0,85. Ce résultat est en accord avec les données obtenues par d'autres auteurs (De Brabander et al 1974 et 1976, Vérité et Journet 1973) dans le cas de fourrages de bonne valeur alimentaire. Il se confirme ainsi que l'encombrement des betteraves fourragères s'accroît avec la qualité du fourrage auquel elles sont associées. Malgré cette substitution élevée, le niveau des apports énergétiques de la ration a été amélioré d'environ 1 UFL grâce en particulier à l'importance de l'écart $(0,40 \mathrm{UFL} / \mathrm{kg} \mathrm{MS})$ entre les concentrations énergétiques du trèfle violet et des betteraves. Dans nos conditions d'utilisation, les betteraves fourragères représentant une faible proportion de la ration ont de ce fait été valorisées à une concentration énergétique voisine $(1,10$ à 1,15 UFL.kg MS) de celle proposée dans les nouvelles tables de l'INRA (1988). Cette introduction a permis d'améliorer le niveau de production permis par la ration de base d'environ $2,5 \mathrm{~kg}$ de lait.

- Cette comparaison a cependant été réalisée à mêmes niveaux de complémentation énergétique et azotée par rapport à la ration de base comportant les mêmes fourrages. L'utilisation des betteraves devrait plutôt être envisagée comme pouvant permettre une réduction des apports d'aliments concentrés. Il serait aussi opportun de les comparer à une autre source énergétique de glucides telle que l'amidon. Cette dernière est en effet facile d'emploi et très disponible à l'heure actuelle par suite de la surproduction de céréales. Cet essai sera entrepris dès 1988 sur le même site expérimental en effectuant une comparaison iso-énergétique et azotée entre les betteraves fourragères et le blé en complément d'une ration mixte d'ensilages de maïs et d'herbe.

\section{Remerciements}

Nous tenons à remercier l'ADBFM de son concours technique qui a permis la réalisation de cet essai.

\section{Références bibliographiques}

DE BRABANDER D.L., AERTS J.V., BOUCQUE C.H., BUYSSE F.X., MOERMANS R.J., 1974. Influence spécifique des betteraves fourragères sur l'ingestion chez les vaches laitières. Revue de l'Agriculture ${ }^{\circ}$ 6, 1479-1491.

DE BRABANDER D.L., AERTS J.V., BOUCQUE C.H. BUYSSE F.X., 1976. Influcence spécifique de betteraves fourragères sur l'ingestion chez les vaches laitières (II). Revue de l'Agriculture $n^{\circ}$ 3, 593-606.

DEDENON N., LE GALL A., MAURIES M., 1988. Ensilage de maïs + ensilage de trêfle violet : plus de lait, moins de taux. Annuel pour l'Eleveur de bovins, 125-130.

ESPINASSE R., 1986. L'ensilage de trèfle violet. Elev. Rentabilité $213,8-9$.

FAVERDIN Ph., HODEN A., COULON J.B., 1987. Recommandations alimentaires pour les vaches laitières. Bull. Tech. CRZV Theix INRA, 70, 133-152.

HODEN A., 1985. Utilisation de la betterave fourragère par les vaches laitières. Document ADBFM, CR de la journée du 12 décembre, Rennes p. 97-104.

HODEN A., COULON J.B., DULPHY J.P., 1985. Influence de l'alimentation sur la composition du lait. 3 - Effets de régimes alimentaires sur les taux butyreux et protéiques. Bull, Tech. CRZV Theix, INRA, 62, 69-79.

HODEN A., MARQUIS B., de la FOYE F.X., 1987. Ensilage de maïs et de trèfle violet pour vaches laitières. Bull. Tech. CRZV THEIX, INRA, 67, 33-37.

HODEN A., COULON J.B., FAVERDIN Ph., 1988. Alimentation des vaches laitières, Chap. 8 ; in «Alimentation des bovins, ovins et caprins". Ed. R. Jarrige. INRA Publications, Route de Saint-Cyr, 78000 Versailles.

INRA, 1988. Alimentation des bovins, ovins et caprins. Ed. R. JARRIGE : INRA Publications, Route de Saint-Cyr, 78000 Versailles.

JOURNET M., CHILLIARD Y., 1985. Influence de l'alimentation sur la composition du lait - 1 - Taux butyreux: facteurs généraux. Bull. Tech. CRZV Theix, INRA, 60, 1324.

KERJEAN J.R., 1988. Du lait fromager pour une grande région fromagère. A la pointe de l'élevage, 193, 15-18.

KEROUANTON J., 1982. Betterave : la relance. A la pointe de l'élevage $\mathrm{n}^{\circ} 135$ et 138.

KEROUANTON J., 1988. Spécial bovins. $\mathrm{N}^{0}$ hors série, A la pointe de l'élevage. Mars.

REMOND B., 1984. Effets du stade de lactation et de l'âge sur la composition du lait. CR Journées INRA, ENSAR, INAPG, Rennes 26-28 septembre.

REMOND B., 1985. Influence de l'alimentation sur la composition du lait de vache. 2 - Taux protéique : facteurs généraux. Bull. Tech. CRZV Theix, INRA, 62, 53-68.

VERITE R., JOURNET M., 1973. Utilisation des quantités élevées de betteraves par les vaches laitières: étude de l'ingestion, de la digestion et des effets sur la production. Ann. Zootech. (22), 219-235. 